\title{
Exploring the Past with Google Cardboard
}

\author{
Adeola Fabola*, Alan Miller*, Richard Fawcett ${ }^{\dagger}$ \\ ${ }^{*}$ School of Computer Science, ${ }^{\dagger}$ School of Art History \\ aef6, alan.miller, rf41@st-andrews.ac.uk \\ University of St Andrews, United Kingdom
}

\begin{abstract}
Combining digital reconstruction with mobile technologies has the potential of enriching visitors experience to historic sites. Through designing a mobile App with Google Cardboard it is possible to use technology already in peoples' pockets to provide immersive on-site exploration of historic sites. This paper looks at our experience in developing such a mobile App which acts as a digital guided tour of the remains of St Andrews Cathedral. The App brings together traditional media such as audio, images, panoramas, $3 D$ video and $4 \pi$ Steradian (or $360^{\circ}$ ) video with a mobile smartphone and Google Cardboard to provide a tour of one of Scotland's most important historic sites. The mobile App is available from both Google Play and iTunes, providing direct delivery to a potential audience of millions. It complements the location-aware mediaeval St Andrews App, which provides a guided tour to the town of St Andrews as a whole. In the absence of Google Cardboard the App is still useful providing both visual content and audio commentary on this historic monument.
\end{abstract}

Index Terms-Panorama, Google Cardboard, Stereoscopic

\section{INTRODUCTION}

Advancements in computer graphics have facilitated the production of 3D digital reconstructions of heritage sites and artefacts. An example of such endeavours is the 3D reconstruction of the St Andrews Cathedral as it stood in the 14th Century using OpenSim software by the Open Virtual Worlds Group at the University of St Andrews (see [1]). This and many more reconstructions were produced with high end computer graphics software and are consequently resourceintensive, having high memory and processor speed requirements. While these reconstructions offer valuable potential in cultural heritage applications, the high computing requirements limits the use cases for these 3D models to demonstrations on powerful computers in fixed environments.

A desirable use case for these models would be to make them accessible on mobile devices. One way this can be achieved is to use pervasive technologies to showcase the 3D models in a lightweight, yet engaging manner, like stereoscopic panoramas that represent $360^{\circ}$ views of the virtual reconstructions. This ongoing work leverages the power of open-source frameworks and web technologies such as WebGl to create a system that can run smoothly in web browsers and on mobile devices. A significant feature of this system is the implementation of stereoscopy to provide the illusion of depth and the incorporation of a light-weight Virtual Reality (VR) headset - the Google Cardboard - to provide an immersive experience.

The outcome of this work is a relatively cheap, lightweight, accessible yet powerful setup that will imbue the general public with the ability to appreciate cultural heritage information using mobile devices and VR headsets such as the Google Cardboard, with the option of an improved VR experience using more powerful PCs and more sophisticated Head Mounted Displays (HMDs) like the Samsung Gear VR.

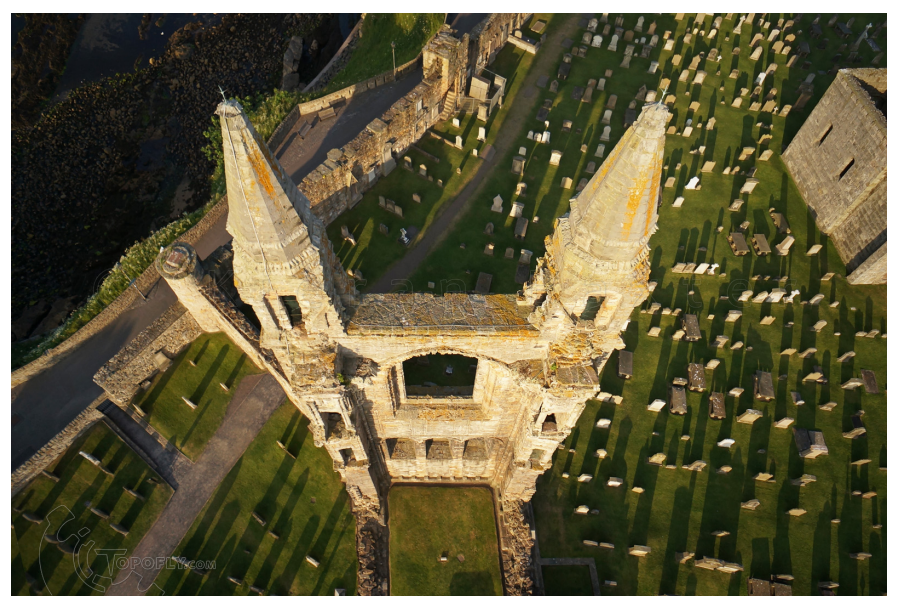

Fig. 1: View of St Andrews Cathedral Today. Source: Kieran Baxter

The remainder of the paper is organized as follows. In Section I-A a background discussion on the history of interpretation mechanisms in the context of cultural heritage is presented. Section III provides a discussion of related work and highlights some challenges associated with on-site, mobile exploration of heritage sites. Section III introduces the design and details the user experience and interaction paradigms of the system. The steps taken to implement and evaluate the system are outlined in sections IV and V respectively, and section VI concludes the paper and provides a discussion of some challenges and future work.

\section{A. History of panoramas in cultural heritage}

Panoramas have existed in different forms such as Marcorama, Cineorama, Stereorama, Diorama and Georama since the eighteenth century, and have been used to interpret and represent cultural heritage information. The earliest panoramas were resource-intensive to setup and were limited in content; although they represented an improvement on the state of the art, the emergence of film caused them to fall into decline in the early twentieth century [2].

Panoramas have made a comeback in recent decades and this resurgence can be attributed to three main factors [2] p.308], the most pertinent to this work being the desire to engage in virtual time travel (to visualise the past, for example) and virtual space travel (as a substitute for real-life travel). This has led to the emergence of numerous panoramic applications in virtual heritage systems. These systems take on different forms such as installations that surround the user, panoramic images viewed on computer screens with the aid of specialised programs (e.g. Apples QuickTime Virtual Reality player) and web-based applications [2].

Efforts have been made to improve on the immersive experience offered by panoramas in virtual heritage systems [2] 


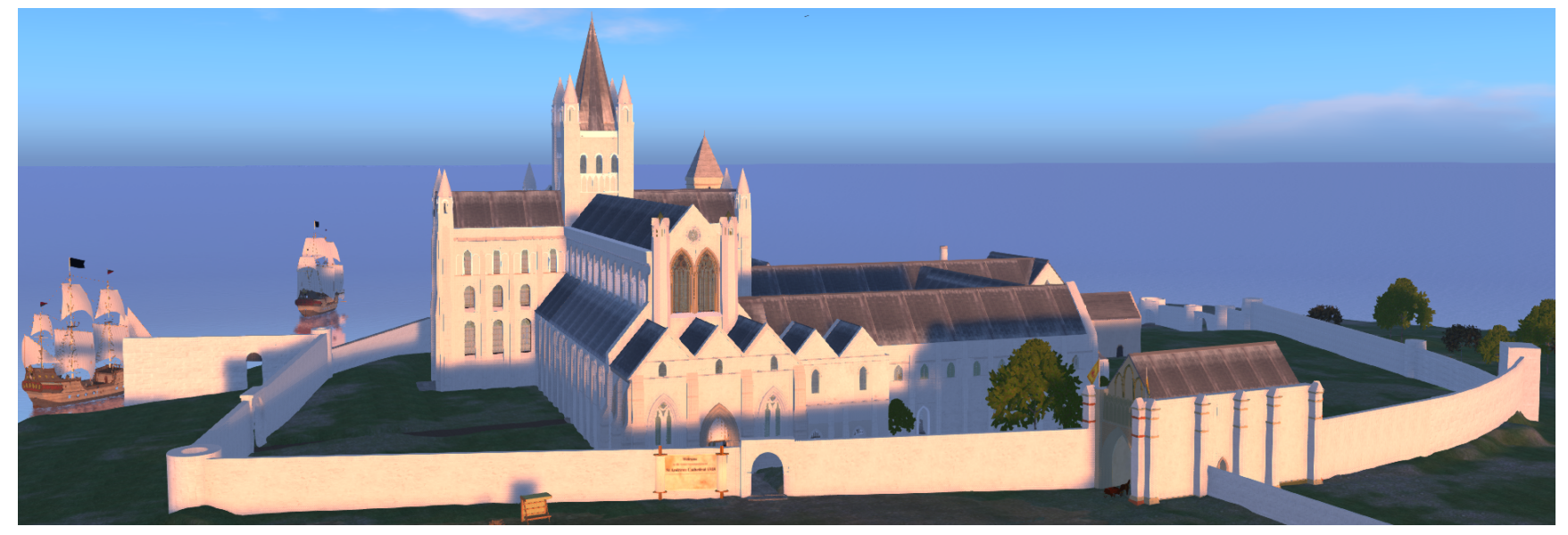

Fig. 2: Digital Model of St Andrews Cathedral, Viewing point slightly elevated to the North West

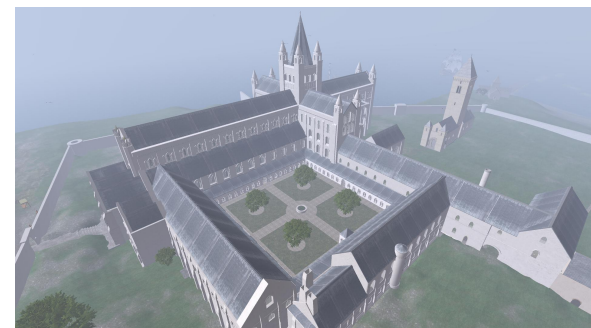

Fig. 3: Aerial view of the Cloister

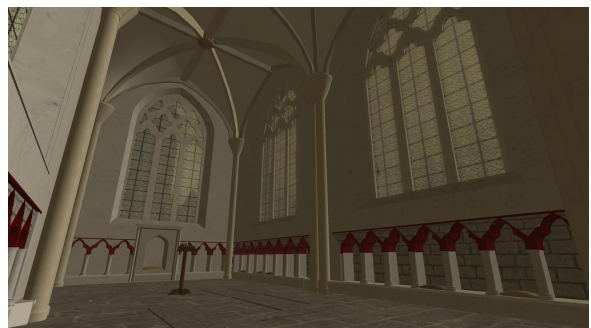

Fig. 4: Reticulated Windows in the Chapter House

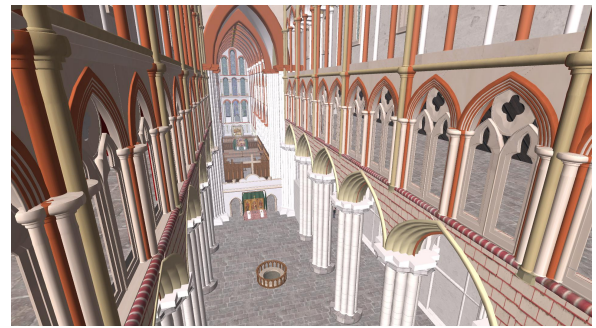

Fig. 5: Internal view from Naive looking East using different approaches such as stereoscopy and HMDs. The advent of light-weight VR headsets such as the Google Cardboard opens up new possibilities for disseminating cultural heritage information, and this serves as the motivation for this work, with the St Andrews Cathedral in Scotland as the subject matter.

\section{B. St Andrews Cathedral}

St Andrews Cathedral represents an important figure in Scottish history; it was the largest church in Scotland and was home to eminent religious figures and activities in its prime [1]. Significant fires and the Scottish reformation heralded a series of events that caused the Cathedral to gradually descend into ruins, which still constitute a popular tourist attraction to this day (see Fig 1).

Having recognised the importance of the St Andrews Cathedral in the history of the town and Scotland as a whole, Kennedy et. al. [1] developed a 3D reconstruction of the Cathedral as it stood in 1318 using open source, virtual worlds technology, thus providing an avenue for users to experience the Cathedral through readily-available computer technologies. The reconstruction process was based on previously-conducted research on medieval Scottish history. This served to provide contextual input, with Ordinance Survey data, architectural plans and ancient drawings providing input for the visualisation of tangible elements of the Cathedral. Non Player Characters (NPCs) - avatars programmed to respond to user interaction and predefined events - were embedded in different sections of the model to improve on the interactive experience of the users. The 3D model of the Cathedral (see Figs 2 5) has been adopted in several use cases such as exhibitions, guided tours, and teaching at different educational levels; it

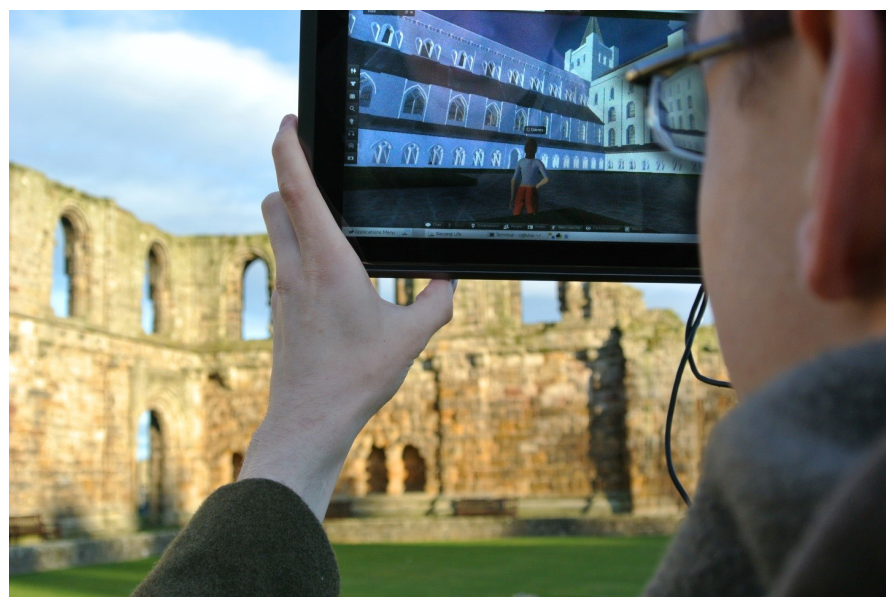

Fig. 6: Tablet running a virtual world viewer. Source: 3]

also serves as an educational tool which is freely accessible over the Internet [1].

\section{RELATED WORK}

Davies et al. [3] details the design and implementation of a Virtual Time Window (VTW) system that facilitates on-site, mobile exploration of heritage sites with a tablet running a virtual world viewer connected wirelessly to an OpenSim server on which the virtual reconstruction of the Cathedral is hosted (see Fig 6). The system uses GPS data to facilitate location tracking as well as sensors (accelerometer and magnetometer) to synchronise the user's position in the real world with the avatar's position in the virtual environment, without the need for explicit interaction (i.e. using input devices) on the part 


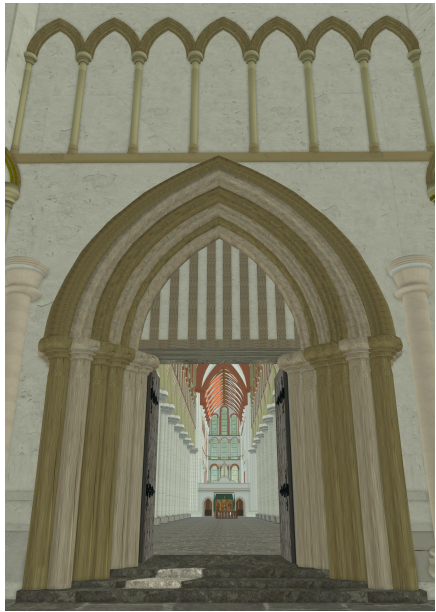

Fig. 7: West Door as it stood in 1318

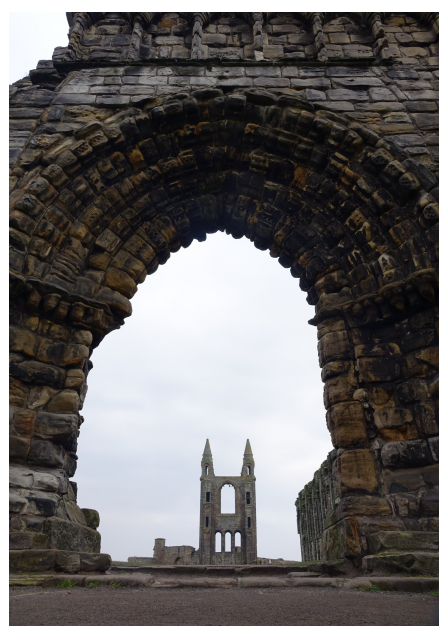

Fig. 8: Remains of the West Door of the user, thus providing a solution to the vacancy problem while keeping cognitive load to a minimum.

Their approach revealed a number of challenges. For instance, the accuracy of GPS tracking constituted a technical challenge as the translation of real world coordinates to coordinates in the virtual environment (OpenSim) is central to the operation of the system. Also, it was observed that typical mobile devices do not currently have the capacity to run both the virtual world client and server; this necessitated a dedicated server to host the reconstructed model, to which the client was connected over a wireless network. This had performance implications in terms of the wireless coverage required to maintain a connection to the server and the framerate obtainable on the tablet in rendering the models in the virtual environment. In addition to these technical challenges, a usage issue was observed, as the system was designed to be used by holding up a tablet while moving around a site. An issue with this approach is the hampered vision that may arise from the glare of the sun on the screen, which may affect user experience. Consequently, this work details the design and implementation of a system that proposes to eliminate (or at least mitigate) the aforementioned challenges.

\section{APPLICATION}

To provide novel means for the interpretation of cultural heritage information, a mobile application was developed to take advantage of the pervasive nature and increasing computing power of mobile technologies. To provide an engaging experience, stereoscopy was incorporated into the system and the Google Cardboard [4] (shown in Fig 13) was leveraged to produce a light-weight, virtual reality system - an app that provides an immersive experience without the encumbrances and cost implications of venerable VR headsets like the Oculus Rift [5]. The features and interaction paradigms of the system are discussed in the sections that follow.

\section{A. Locations}

Ten locations in and around the Cathedral are represented in the System. Fig 15 shows the geographical location of each one as viewed from the top of the Cathedral. When a user begins the tour, the locations are traversed in turn (starting at the Pends and ending at the Refectory), with users going from one location to the next. This facilitates on-site exploration of the Cathedral because at each location, users can hold up the viewer to observe the reconstructed view of the Cathedral, and then compare this with the Cathedral's current state (see figs 7 - 10. This enables a comparison of the remains of the Cathedral with a view of its majestic architecture as at 1318 . The significance of these locations are highlighted below:

Pends: Located on the South-west of the Cathedral, it was one of at least four gates into the Cathedral precinct. It was important for the gates to be as impressive as possible to give an idea of the high standing of the Augustinian community within the precinct.

West Door: The great processional entrance into the Cathedral. It was originally intended to be two bays further west, but a storm in the 1270s blew down the west front before it was completed, causing it to be relocated two bays further east. It is pertinent to note that it was not the general entrance for people visiting the Cathedral, as that was on the North side of the Nave.

Nave: The largest space within the cathedral. It was originally intended to be fourteen bays long but owing to the storm in the 1270s, it was reduced to twelve bays in length, but still remained largely the largest Nave of any Cathedrals built in Scotland.

Transept: The cross-arms of the church. On the east side of each, there were three chapels which together contained six of the many altars that were spread throughout the Cathedral. They had a symbolic purpose of giving the Cathedral the cross shape that was a reference to the cross of Christ.

Choir: Contained the stalls for the canons, where they sat to observe the daily services and masses. It was originally at the far east end of the building but was later cut off to create a relic chamber to hold the bones of St Andrew and other saints. Relics: Located east of the transept, in arguably the most important part of the Cathedral. It was originally intended to contain the high altar but was later adapted as a chamber to hold the relics of St Andrew and other saints.

Cloister: A square-shaped, open space on the South side of the Cathedral, surrounded by covered walkways and various buildings to accommodate the daily life of the canons. The North side of the cloister was often used by the canons to observe their daily readings and (in good weather) copying of manuscripts.

Chapter House: The second most important building in the monastery, with very impressive interior. It was the business room of the priory where the priors and canons discussed their business needs. It was also where the canons were expected to confess their faults.

Warming House: Underneath the South-end of the dormitory, it was covered by stone vaulting with a large fireplace. It was the location where the canons spent most of their time in cold weather, as it was the only social fireplace allowed by rule in the Cathedral.

Refectory: The third most important building in the monastery, where the canons took their meals each day. It was located on the first floor of the Cathedral, in reference to the upper room where Christ and His disciples took the last supper.

\section{B. User Experience}

This section highlights the main features of the system and how these features contribute to the user experience. 


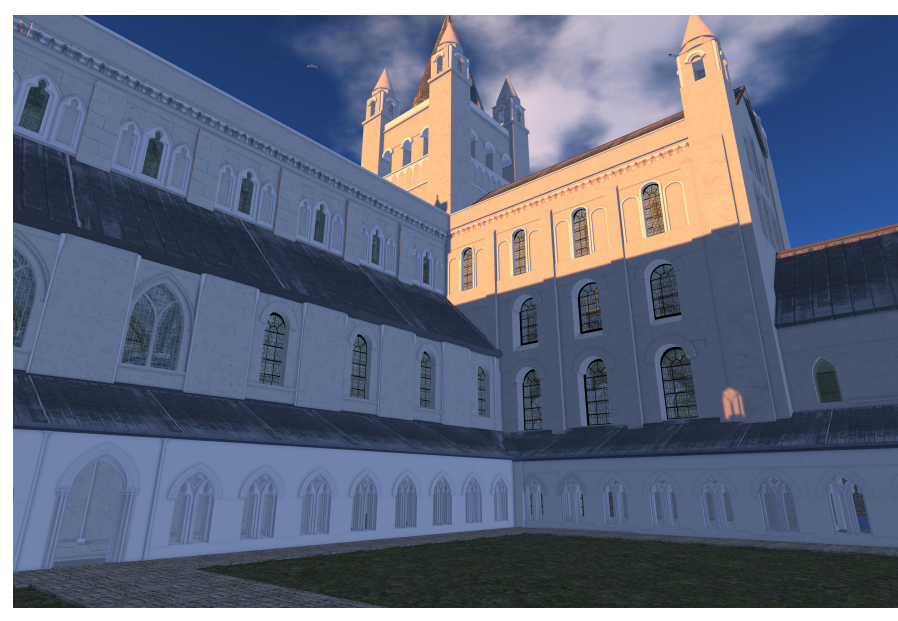

Fig. 9: Cloister as it stood in 1318

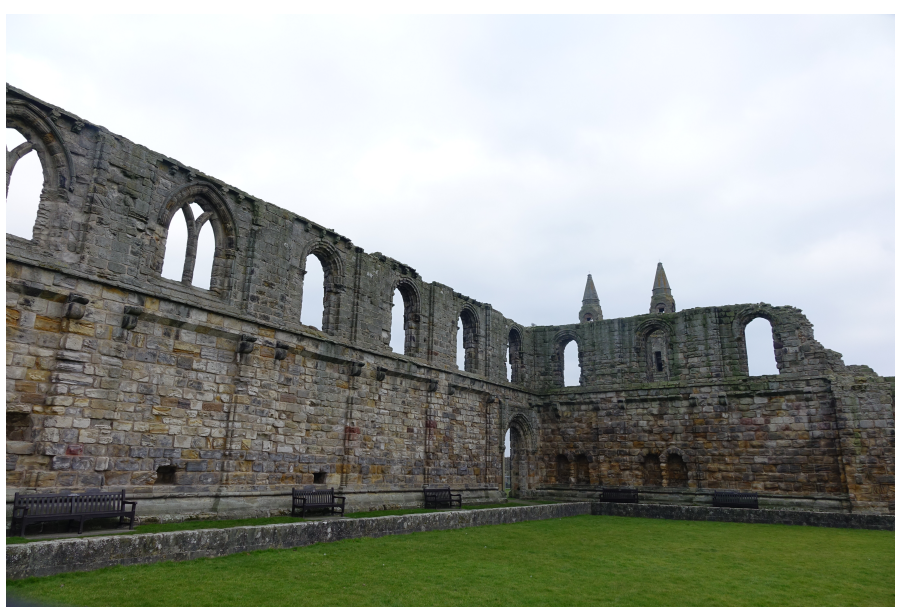

Fig. 10: Cloister at the remains of the St Andrews Cathedral

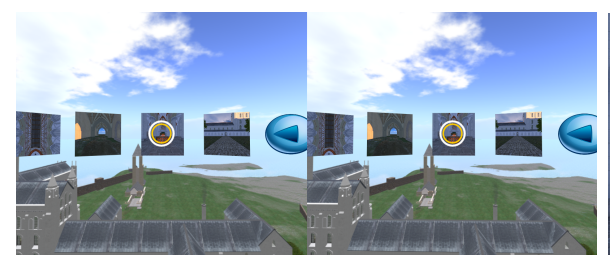

Fig. 11: Top level menu for visual trail

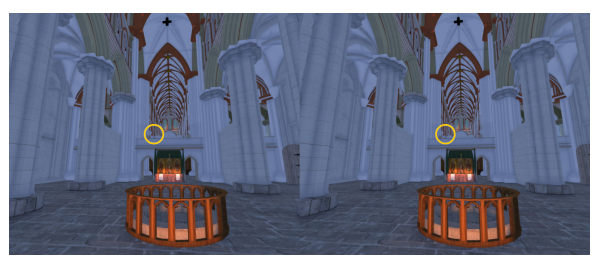

Fig. 12: Trail location: Cathedral Nave

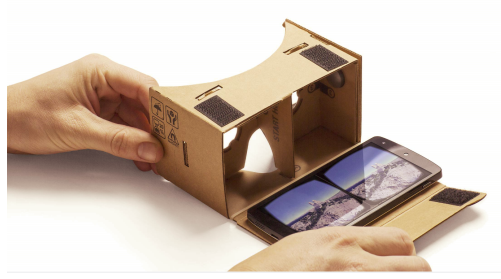

Fig. 13: Google Cardboard. Source: 4
1) Visual Trail: The system features several stereoscopic $360^{\circ}$ image panoramas, where each panorama corresponds to a location (see section III-A) that is linked to other panoramas to form a virtual tour of the Cathedral (see Fig 12). The locations also have associated audio narratives that provide an engaging experience to the user. When a user navigates to a new location, the associated audio plays in the background, thus providing more information about the location and situating it within the context of the tour.

2) Audio Narratives: In addition to the audio narratives, the system features audio clips that provide more information about the Cathedral. These clips detail the significance of the different locations, as narrated by domain experts based on years of scholarly research.

3) Videos: Videos of the 3D model of the Cathedral are also featured on the system. There are two types of videos included: stereoscopic fly-though videos, which provide an illusion of depth with limited interaction (pause and play) and $4 \pi$ Steradian videos, which provide an improved immersive experience by situating users in the middle of an environment with a $360^{\circ}$ panoramic view. Details of how these videos were produced and incorporated into the system are provided in Section IV

4) $3 D$ objects: Embedded within each location are 3D objects (or hotspots) which can be triggered to perform actions such as navigating to a new location, launching a stereoscopic video or launching a popup with more information.

\section{Navigation}

The integration of the app with the Google Cardboard invalidates the default interaction mechanism of most mobile apps (where users tap or scroll the screens of their mobile devices with their fingers to initiate actions) and thus necessitates the development of a hands-free interaction paradigm. For this reason, two hands-free modes of interaction were implemented and provided as options to users.

The first interaction mode - the manual mode - leverages the head-tracking features of virtual reality headsets.This interaction mechanism is implemented on the app by means of a selector which is placed in front of the camera and hence always in the users view. The selector animates (grows and shrinks) when placed on a hotspot, and after a few seconds of continuous animation, the hotspots associated action is triggered (see Fig 11). This interaction paradigm involves placing the selector on hotspots or looking towards specified directions to trigger events. For instance, users can navigate back to the starting point by looking in the upward direction for a few seconds.

The second mode of interaction - the location-based mode - leverages the inbuilt GPS feature of mobile devices. In this mode, the system periodically polls the user's device for its latitude/longitude coordinates, compares these latitude and longitude values to those of the different locations present around the site, and triggers a navigation effect when the user is within range of a site location. For instance, while exploring the Cathedral, as a user gets within range of the Pends (for example) the system provides a notification alerting the user to the new location detected and then navigates to the location in the virtual environment. It is pertinent to note that the manual mode of interaction is always enabled; however users can enable or disable the location-based mode so as to make the exploration experience less disruptive. 


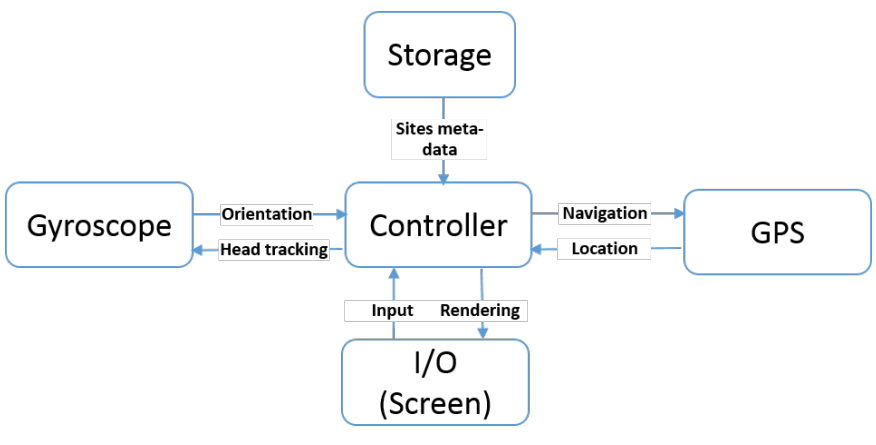

Fig. 14: High level architecture of the system

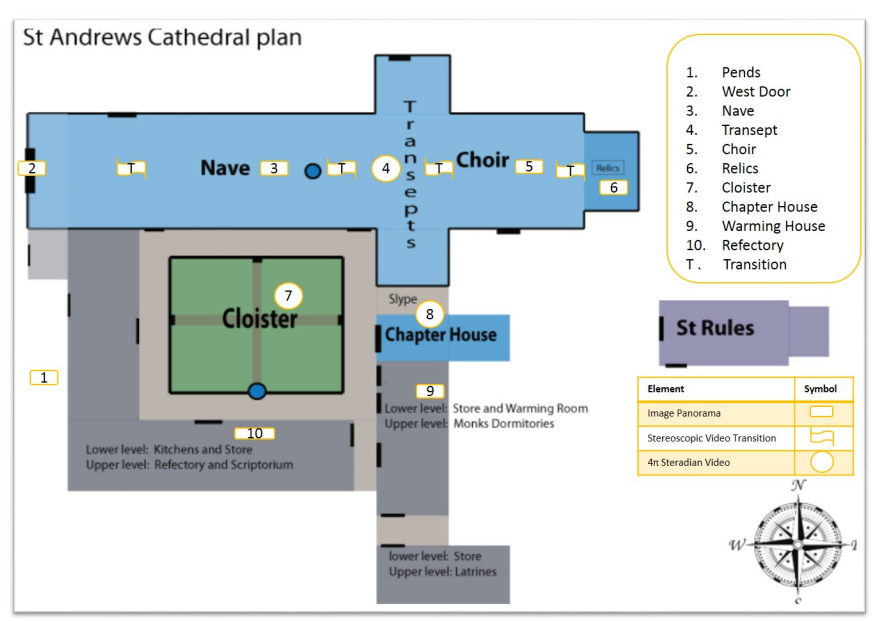

Fig. 15: Plan of St Andrews Cathedral

\section{System ImPlementation ANd ARChitecture}

Fig 15 shows the floor plan of the Cathedral. The locations on the tour are shown with different elements to distinguish between their features. Locations such as the Cathedral Pends and West Gate were represented with image panoramas (shown as rounded rectangles) to provide $360^{\circ}$ panoramic views. The Transept, Chapter House and Cloister were represented with dynamic, $4 \pi$ Steradian time-lapse videos (shown as circles) to highlight the effects of lighting and shadows on the architectural elements of the building. Stereoscopic videos (shown as punchtapes) were used to provide transition effects between locations. For instance, when moving from the West Gate to the Nave, and from the Nave to the Transept, these videos were used to provide the illusion of movement along the halls of the Cathedral.

\section{A. Conceptual Model}

The system was implemented as an application that runs on mobile devices, hence the features tap into components that are bundled with smartphones. Each location (or scene) is rendered as a $360^{\circ}$ image or video panorama (with accompanying audio narratives); these multimedia resources are accessed from the devices storage to facilitate quick read operations. The devices GPS is used to detect the latitude and longitude coordinates of the user (which are compared to the coordinates of the different locations) to facilitate the location-based navigation. Head tracking is enabled by means of the devices gyroscope.
As the user turns their head to look around a location, the system reads the pitch, yaw and roll values from the gyroscope and updates the view on the screen. This process takes place quick enough (ideally at 60fps) to prevent lag/latency in head tracking. The high level architecture of the system is shown in Fig 14

\section{B. System Workflow}

The steps taken to implement the components of the system are discussed in the sections that follow.

1) Stereoscopic Image panoramas: The implementation of the image panoramas featured on the system is discussed below:

a) Creating a $360^{\circ}$ image panorama viewer: Several approaches exist for representing panoramas including spherical, cylindrical and cubic [6]. The spherical (or equirectangular) approach produces a panorama out of single image with an equirectangular (or mercator) projection which is mapped onto the surface of a sphere. The cylindrical approach produces a panorama out of a series of vertical image strips that form a $360^{\circ}$ view of the environment to be represented, with the strips mapped onto the surface of a cylinder. The cubic approach produces a panorama out of six images with a 1:1 aspect ratio, where each image is mapped to one side of a cube (hence the name) with the viewer at the centre. The cubic approach was adopted owing to its computational simplicity [6] and convenience (capturing the six images required for a cubic panorama proved much easier in the development environment than producing an equirectangular image which represents a $360^{\circ}$ view of the scene to be rendered).

As the system was implemented using web-compliant technologies, an open-source JavaScript library [7] was employed in the development of the image panorama viewer. To create the cubic panoramas, several images were captured (using a virtual worlds capture tool [8]) from different locations of the Cathedral 3D model. The capturing tool was set-up to capture six images (the front, back, right, left, up and down views) for each location (see Fig 16 . The panoramas were then rendered by creating a scene, putting a camera at the centre of the scene, and then adding the six images to the scene with each image having specific position and rotation values so as to represent the six faces of a cube. The outcome of this exercise was several $360^{\circ}$ image panoramas that facilitate traditional navigation (panning, zooming, and so on) in a web browser. Having developed this, stereoscopy was incorporated into the panorama viewer to provide the illusion of depth.

b) Incorporating stereoscopy into the panorama viewer: The next step towards providing an immersive experience in the system was to create an illusion of depth. Research conducted revealed a number of techniques to do this, one of which is known as stereoscopy - a technique that provides the illusion of depth by creating two images (one for each eye) with slightly different perspectives [9]. Stereoscopy was incorporated into the panorama viewer by providing two sideby-side views (one for each eye) and using the CSS 3D transform property to produce a perspective offset that simulates the Interpupillary distance (IPD) - the distance between the centre of the two eyes. 


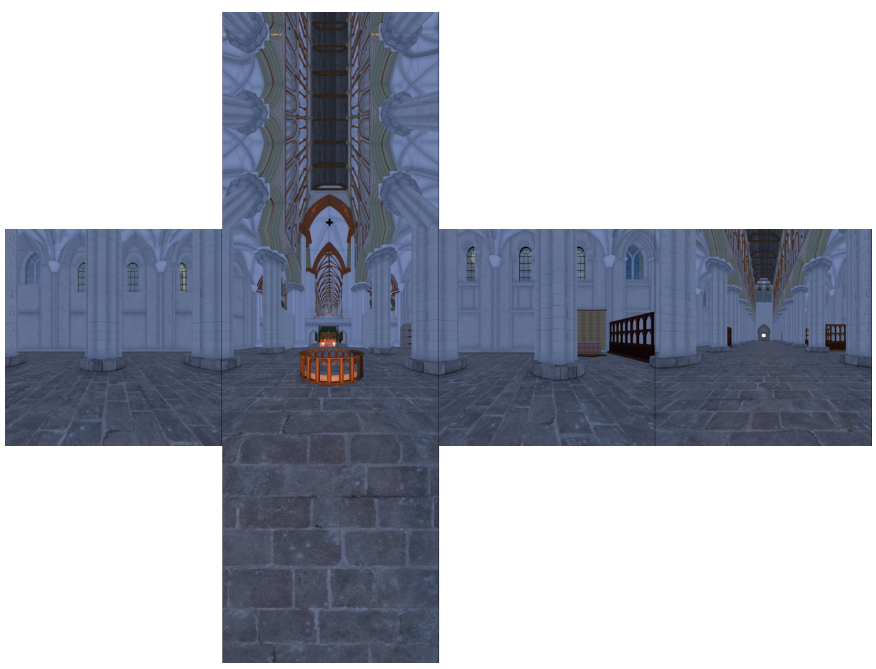

Fig. 16: Six faces of a cubic panorama representing the Nave

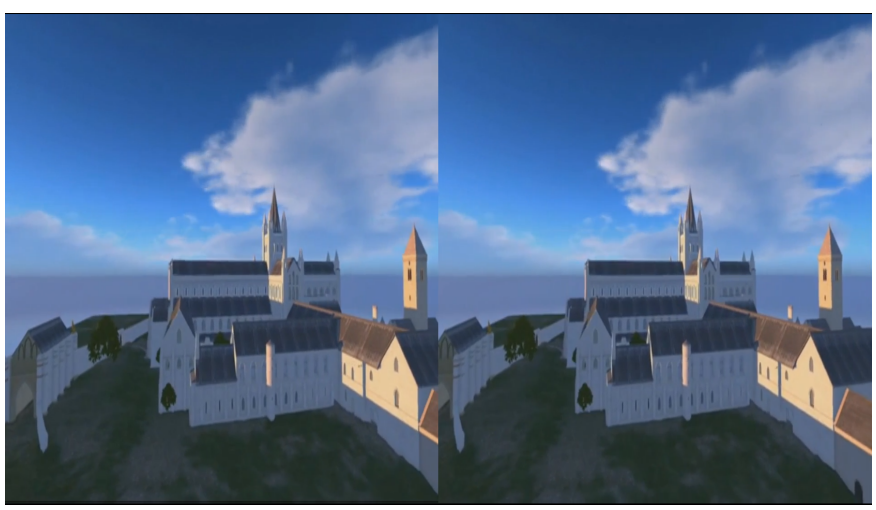

Fig. 17: Stereoscopic video of the Cathedral.

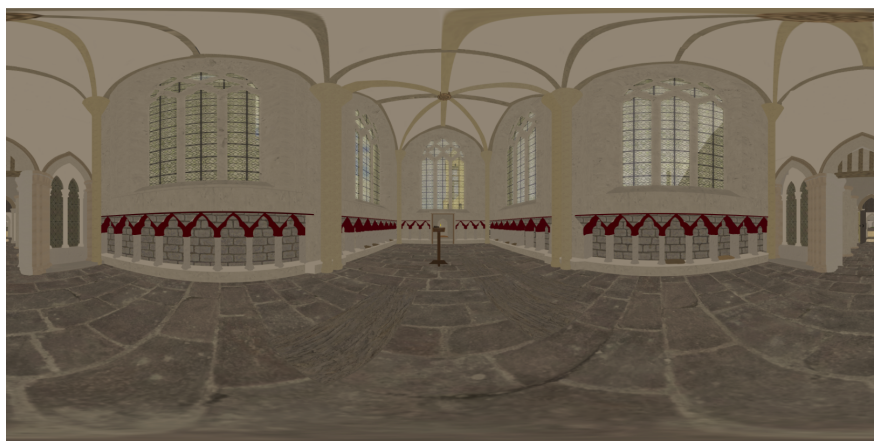

Fig. 18: Equirectangular projection of the Chapter House.

c) Adapting the viewer for mobile and Virtual Reality (VR) platforms: To adapt the stereoscopic panoramas for use on mobile devices, the Google Cardboard [4] was leveraged to produce a light-weight, virtual reality system - an app that features a strong immersive experience without the encumbrances and cost implications of venerable VR headsets like the Oculus Rift [5]. An integral feature of VR headsets is a suite of sensors - gyroscope, magnetometer and accelerometer - that track the users orientation and position in space. A cursory inspection of the Google Cardboard makes it obvious that it is not endowed with sensors to facilitate head tracking; however, modern smartphones are, hence the combination of a modern smartphone and the Google Cardboard makes possible the development of immersive applications that detect users orientation information and adjust the view accordingly. For the developed system, head-tracking was implemented by detecting changes in the devices gyroscope parameters and adjusting the users view. For instance when users move their heads (and by extension, their devices), the difference between the current and previous positions is read from the gyroscope and the camera view is updated by a measure of this difference. By virtue of this, a web application adapted for use with the Google Cardboard on mobile devices was implemented.

2) Stereoscopic Videos: Existing footage of the 3D model of the Cathedral were transformed into stereoscopic form and then incorporated into the system. The transformation process entailed loading the video into a video editing software [10], rendering two side-by-side views (one for each eye), and then varying the perspectives of the two views by cropping out a portion of the right and the left views of the video for the left and right eyes respectively, to produce an offset that simulates the Interpupillary distance (IPD). The video (snapshot shown in Fig 17) was then embedded into the stereoscopic panorama viewer as a hotspot that can be triggered by placing the selector on it for a few seconds.

3) $4 \pi$ Steradian video panoramas: The system features $4 \pi$ Steradian video panoramas (usually referred to as $360^{\circ}$ videos) that possess all the features of the image panoramas, with the added benefit having dynamic content (i.e. moving elements in videos as opposed to static elements in images). This represents a step-up from traditional panoramas in the sense that most panoramas consist of static image elements. The creation of video panoramas injects dynamism into the scene as users find themselves surrounded by moving objects thus providing an improved immersive experience over traditional panoramas. An example of such videos in the system is a $4 \pi$ Steradian time lapse video of the interior of the Cathedral Chapter House (shown in Fig 18. To create this video, the virtual environment was set-up to go through a full day cycle (morning, afternoon, night with the sun and moon at different positions through the day). The virtual world capture tool [8] was then set-up to capture six images (the front, back, right, left, up and down views) periodically at different times of the day, and then stored in appropriately-named folders. This produced six sets of images for the different times of the day. Each of these six images (which represent the elements of a cubic image panorama) were then converted programmatically into an equirectangular image using an open-source tool [11]. This produced several equirectangular images (one of which is shown in Fig 18) for the different times of the day, which were then processed as frames into an equirectangular video. This video was then mapped onto the surface of a sphere in a scene with a camera at the centre. The outcome of this exercise was a $4 \pi$ Steradian time lapse video that situates users at the centre and facilitates head tracking thus providing an immersive experience.

4) Packaging the system: Having designed a web application for use on mobile devices with support for the Google Cardboard, a framework [12] was leveraged to compile the web-based code (written in HTML5, JavaScript and CSS) into native code for mobile platforms (such as Android, iOS, Windows Mobile and so on) so as to make it a self-contained (offline) mobile application that could be downloaded and in- 
stalled on users devices. This approach offers a write-once-runeverywhere benefit by facilitating the deployment of generic web-based code to several platforms. In addition, deploying the system as a mobile application provided the possibility of distributing it via app stores (iTunes and Google Play) and made it available for use offline (without the need for Internet access) thus providing greater reach and access.

\section{System EVAluation}

Working prototypes of the system have been showcased at cultural heritage gatherings. One of these is the ongoing Fife Pilgrim Way project (http://fifecoastandcountrysidetrust.co.uk/ Fife-Pilgrim-Way_68.html), where the system is being used as a means of digital interpretation. Also, the system framework was adapted to develop a tour for the CAEN township and this was showcased at the Timespan True North Conference (http://timespan.org.uk/true-north-conference/). The system was also demonstrated to stakeholders from the National Trust for Scotland (NTS; http://www.nts.org.uk/Home/), and at several structured workshops such as at TEDXGlasgow 2015 (http://www.tedxglasgow.com/).

The system was tested on both the Android (Google Nexus 7) and iOS (iPhone5) platforms. An interesting comparison emerged, as some of the system features worked differently on both platforms. For instance, while the system features worked satisfactorily on both platforms, the image panoramas appeared smoother on the iPhone5 than the Nexus 7, while the $4 \pi$ Steradian time lapse video panoramas appeared smoother on the Nexus 7 than the iPhone5. These differences in system performance can be attributed to the varying levels of support for WebGL and 3DCSS on Android and iOS devices.

A study was conducted to evaluate users perception of the system. The metrics of interest were the system's (1) usability, measured using the System Usability Scale (SUS) proposed by Brooke [13], and (2) usefulness, defined as the extent to which the system can contribute to the users knowledge of the given subject matter (i.e. St Andrews Cathedral); this was measured using a custom, Likert-scale questionnaire.

Nine (9) participants between the ages of 18 and 44 were recruited for the study by reaching out to colleagues through word-of-mouth and email, and responders were screened to ensure adequate visual, motor and English language competencies to ensure that these do not skew the findings of the study. One participant reported moderate prior experience with virtual reality and HMDs, while all the others reported minimal prior experience. Each evaluation exercise was carried out in a conducive environment (such as participants workplaces, common areas and lounges). Each participant was briefed on the nature and purpose of the evaluation, after which they filled in their demographic details and consented for the evaluation to begin. The participants then embarked on the virtual tour of the $\mathrm{St}$ Andrews Cathedral using the system (while under observation from the researcher) and then filled the questionnaires - the System Usability Scale (SUS) questionnaire, the Educational Value questionnaire and the free-form questionnaire. All nine participants completed the exercise and answered all questions. A breakdown of the results is shown in Fig 19 and a summary is shown in Fig 20. The average usability score obtained was 85.83 (which puts the system over the 90th percentile rank) and the average usefulness score was 88.89 (indicating that the system was judged to be of high educational value by the participants).

Participants also filled a free-form questionnaire which was designed to elicit responses regarding the overall experience as determined by perceived immersion, mode of interaction with the system and technical features of the system (such as latency, resolution and sound quality). All nine participants reported a really immersive 3D experience and one participant described it as a "very cool system". When asked what features they liked, participants cited the high quality graphics, $360^{\circ}$ videos and the responsive head-tracking. Observation of the participants revealed some unexpected findings, for instance some participants expected to see their body parts (hands and feet) in the virtual environment while some expected to be able to move from one place to another in a manner evocative of real world exploration. In addition, while some participants wished for total immersion in the virtual environment (and by implication a disconnection from the real world), others wished to maintain a level of "presence" in the real world at the expense of full virtual world immersion. It is worth noting that no participant reported any signs of dizziness or nausea when asked about it by the researcher. This holds promise for virtual reality headsets as a viable means for exploring heritage sites without the discomfort (brought on by nausea and cognitive load) that has hitherto been associated with HMDs.

A significant outcome of the studies was evident from participants' comments, as all participants stated that the system has improved their perception of virtual reality. In addition, participants were impressed with the development and maturity of current virtual reality headset technologies, and a few expressed a desire to purchase their own virtual reality headsets. Multiple participants also stated that the developed system would be a valuable tool for museums as well as for education and personal entertainment purposes.

\section{CONCLUSION}

Several works exist in the public domain on the use of mobile devices to facilitate on-site exploration of heritage sites. The combination of a smartphone and a lightweight virtual reality headset like the Google Cardboard opens up new possibilities for on-site exploration of heritage sites and provides an improved user experience, by tackling some of the challenges that have been uncovered in previous works.

The system design (which taps into the inbuilt GPS of smartphones) detailed in this work occludes the need for translating real world latitude and longitude coordinates to virtual world coordinates. As a user explores the site, the system periodically polls the device's GPS for location data, compares the latitude and longitude values to those of the different locations present around the site, and triggers a navigation effect when the user is within range of a site location. This eliminates the overhead that arises from translating real world coordinates to virtual world coordinates and thus constitutes an improvement on performance. In addition, the enclosure of the smartphone in the VR headset provides a level of immersion that may not be available when viewing objects on a device's screen. Furthermore, it makes the system well-suited for onsite exploration of a site, as the casing serves an additional 


\begin{tabular}{|c|c|c|}
\hline Participant & Usability & Usefulness \\
\hline 1 & 92.5 & 85 \\
\hline 2 & 100 & 100 \\
\hline 3 & 77.5 & 90 \\
\hline 4 & 87.5 & 80 \\
\hline 5 & 100 & 100 \\
\hline 6 & 85 & 90 \\
\hline 7 & 95 & 92.5 \\
\hline 8 & 60 & 85 \\
\hline 9 & 75 & 77.5 \\
\hline Average & 85.83 & 88.89 \\
\hline
\end{tabular}

Fig. 19: Participants' usability and usefulness scores

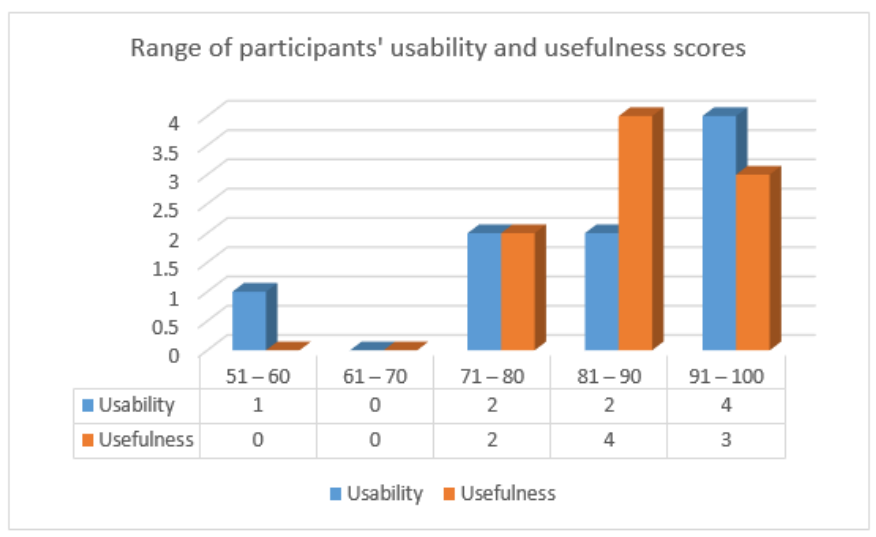

Fig. 20: Summary of participants' usability and usefulness scores

function of eliminating any glare (from the sun) that hampers vision; an issue which may arise when viewing objects on a screen outdoors.

\section{A. Challenges}

The major challenge encountered in the development of the system was the size constraints owing to the images, audio and video files included. The inclusion of high-quality multimedia files significantly increases the storage space required for the overall system to function and this translates directly to a relatively large mobile app size. The implication of this is twofold:

Firstly, it introduces complexities in making the app available on app stores. For instance, the system developed is in excess of $50 \mathrm{MB}$ (MegaBytes), which exceeds the upper limit for apps that can be uploaded to the Google Play store for Android, hence necessitating the use of expansion files.

Secondly, it may put users off from downloading the App from the respective App stores, given the stable Internet connection, time, data and storage allowances required to carry out such downloads. This limitation can be overcome by storing the multimedia resources on an online server (as opposed to on the local device) and retrieving them when required. While this significantly reduces the storage requirements and makes the App much faster to download, it may negatively impact the user experience as users will be unable to use the system while offline and may have to endure lengthy loading times while online.

\section{B. Future Work}

A desired feature of digital heritage systems is the ability to compare the past and present states of the entity under observation. Specifically, it would be interesting to compare the $3 \mathrm{D}$ virtual reconstruction of the St Andrews Cathedral (which constitutes a representation of its past) with the ruins at the site today. This comparison can be facilitated by observing a view of the real world through the device's camera while using the headset. Different modalities can be implemented, such as switching between the real world and virtual world views, augmenting the real world view with virtual world content (or vice versa) and/or blending the two views together. This will facilitate comparisons of the Cathedral's past glory and its present state, thus fostering an appreciation of its status as a tourist attraction.

\section{REFERENCES}

[1] S. Kennedy, et al. "Exploring canons \& cathedrals with Open Virtual Worlds: The recreation of St Andrews Cathedral, St Andrews day, 1318" 2013 Digit. Herit. Int. Congr., pp. 273-280, Oct. 2013.

[2] F. Cameron and S. Kenderdine, "Speaking in Rama: Panoramic Vision in Cultural Heritage Visualization" in Theorizing Digital Cultural Heritage: A Critical Discourse, Cambridge, MA, England: MIT Press, 2007, ch. 15, pp.301-331

[3] C. Davies, A. Miller, and C. Allison, "Mobile Cross Reality for cultural heritage," in Proc. Digit. 2013 - Fed. 19th Intl VSMM, 10th Eurographics GCH, 2nd UNESCO Mem. World Conf. Plus Spec. Sess. fromCAA, Arqueol. 2.0 al., vol. 1, pp. 331-338, 2013.

[4] Google. Google Cardboard [Online]. Available: https://www.google. com/get/cardboard/ [Accessed: 30 March 2015].

[5] Oculus VR. Oculus Rift - Virtual Reality Headset for 3D Gaming [Online]. Available: https://www.oculus.com [Accessed: 30 March 2015].

[6] S. Salehi and E. Dubois, "Alignment of cubic-panorama image datasets using epipolar geometry" in ICASSP, IEEE Int. Conf. Acoust. Speech Signal Process. - Proc., pp. 1545-1548, 2011.

[7] three.js. three.js - Javascript 3D Library [Online]. Available: http: //threejs.org/ [Accessed: 30 March 2015].

[8] J. McCaffery. (2012, Nov. 20). Chimera [Online]. Available http://blogs. cs.st-andrews.ac.uk/mccaffery/2012/11/20/armadillo-proxy/ [Accessed: 30 March 2015].

[9] P. Bourke, "Synthetic stereoscopic panoramic images" in Interact. Technol. Sociotechnical Syst., pp. 147-155, 2006.

[10] Blender Foundation. Features - blenderorg - Home of the Blender project - Free and Open 3D Creation Software. [Online]. Available: http://www.blender.org/. [Accessed: 30 March 2015].

[11] PanoTools.org Wiki. Panorama tools. [Online]. Available: http://wiki. panotools.org/Panorama_tools [Accessed: 30 March 2015].

[12] Adobe Systems Inc. PhoneGap - About. [Online]. Available: http:// www.phonegap.com/ [Accessed: 30 March 2015].

[13] J. Brooke. "SUS-A quick and dirty usability scale" Usability evaluation in industry , pp. 189-194, 1996 